\title{
Dynamic formation of primary grain structures on squared steel billets produced by continuous casting (computer simulation)
}

\author{
A. Ramírez-López ${ }^{1,2}$ • D. Muñoz-Negrón ${ }^{1}$ M. Palomar-Pardavé ${ }^{2}$. \\ M. A. Romero-Romo ${ }^{2} \cdot \mathrm{J}_{\text {. Gonzalez-Trejo }}{ }^{2}$
}

Received: 21 September 2016/Accepted: 12 December 2016/Published online: 20 December 2016

(C) The Author(s) 2016. This article is published with open access at Springerlink.com

\begin{abstract}
The present work is dedicated to explain the development of a computational model for simulating the grain structure formed in a squared billet produced by continuous casting. During steel solidification, three different grain structures are formed as a function of the heat removal conditions. The solidification times previously calculated using a finite difference method were used as input data in order to simulate dynamically the evolution of the grain formation. Computational routines for grouping, counting and classifying have been programmed to evidence the influence of the solidification speed on the grain morphology resulted. Criterions based on solidification speed and time on mushy are used to establish the transitions zones between chill, columnar and equiaxed grains. Routines to simulate grain nucleation and growth based on chaos theory have been included to create a graphical cellular automaton on the computer screen to animate the grain structure formation.
\end{abstract}

Keywords Computer algorithms · Steel solidification · Grain nucleation and growth $\cdot$ Chill $\cdot$ Columnar and equiaxed . Cellular automata $\cdot$ Continuous casting

A. Ramírez-López

adan.ramirez@itam.mx; adaralo@starmedia.com

1 Academic Department of Industrial Engineering, Instituto Tecnológico Autónomo de México (ITAM), Z.P. 01080 Mexico city, Mexico

2 Department of Materials Science, Universidad Autónoma Metropolitana (UAM- Azcapotzalco), Z.P. 02200 Mexico city, Mexico

\section{Introduction}

The solidification structure control is important in continuous casting process $(\mathrm{CCP})$ is due to it may affect the quality and performance of the steel products. It can cause troubles and defects during manufacturing. Many factors affect the quality of casting and it is necessary to understand properly the phenomena occurring in CCP from an industrial and scientific point of view. Solidification is a difficult problem to be treated. Heterogeneity on grain morphology and the geometrical complexity makes necessary the inclusion of Monte Carlo Methods, stochastic procedures and chaos theory. The beginners [1] who tried to simulate grain structures obtained by continuous casting used basic geometrical approaches. Recently the computational models have become more sophisticated and many authors $[2-5,11]$ have developed models based on cellular automata to simulate in better detail grain structures. Some authors have developed models for ferritic and non ferritic materials due to there are different metals and alloys, these authors also have developed models to simulate the dendrite formation; which is the elementary simulation to form a grain. Nevertheless the scales must be reduced. Some other authors $[5-8,11]$ have been working on the development of computational models using cellular automaton and reproduce the multi-grain formation.

The models developed by other authors [9-18] have incorporated physical theory related with the microstructure formation of these materials during solidification. About it, all the models also [2-21] treat the solidification problem as a function of the nucleation and grain growth. Some authors [16] have simulated this process using parallel computing methods. Some of them [12, $15,17]$ treat specific problems such as the interruption of grain growth due to some phases or inclusions the solidification of the melt. The problem about the scales 
for simulation is also treated by some of them including the limits of the graphical representation [18-21]. Some other authors remark the importance of the mushy on the final structure [13, 18-21, 23-27]. The problem of transitions zones is also treated by some authors [21]. Nevertheless the presented work treats the problem of the grain structure formation during solidification of a steel squared billet using information about the heat removal. In consequence the model simulates each solidification case as a function of real quenching conditions previously calculated.

The main purpose of this research is to create a computational algorithm capable to reproduce the grain structure formed during solidification but dynamically using the information of a previous thermal analysis and in addition to prove the influence with mathematical and physical facts of the steel thermal behavior over the grain structure resulted.

The present authors developed a computer simulator for calculating the steel thermal behavior previously [22]. The model was based on the solution of a two dimensional equation system. In which a control volume of the steel was discretizated using 3D elements. Nevertheless due to the energy required for heat the steel $\left(\mathrm{H}_{\mathrm{I}, \mathrm{J}}\right)$ until the casting temperature $\left(\mathrm{T}_{\text {casting }}\right)$ is a numeric value a regular structured squared mesh was used for representing. The equations to simulate heat removal conditions according with the steel position on the continuous casting machine were solved according with the boundary conditions. A finite difference Method known as Cranck Nicholson rule was used to solve the system including radiation, forced convection, conduction and state equations. The 3D elements were managed as nodes where the sub indexes (I) and (J) were used to identify the nodal position on the computational array. In the present work a cellular automata is used to represent each nodal position displaying on the screen every cell with a pixel- color. A cell represents each nodal position as is explained in Fig. 1. The solidification times saved in the computational arrays after thermal calculation are used as data input in order to simulate the grain structure formation according with the dynamical evolution of the solidification.

The nodal times were saved in a file using an ordinate format for making compatible the information with some others software or models. Nevertheless the grain growth model developed in this work does not work with temperatures or enthalpies. Solidification times are load from files in order to confirm the physical principle exposed in Eq. (1) and Fig. 1. This equation represents the equivalent derived from the work with enthalpies and temperatures but also with the times according with the evolution of the solidification process due to the incorporation of a monitoring procedure and

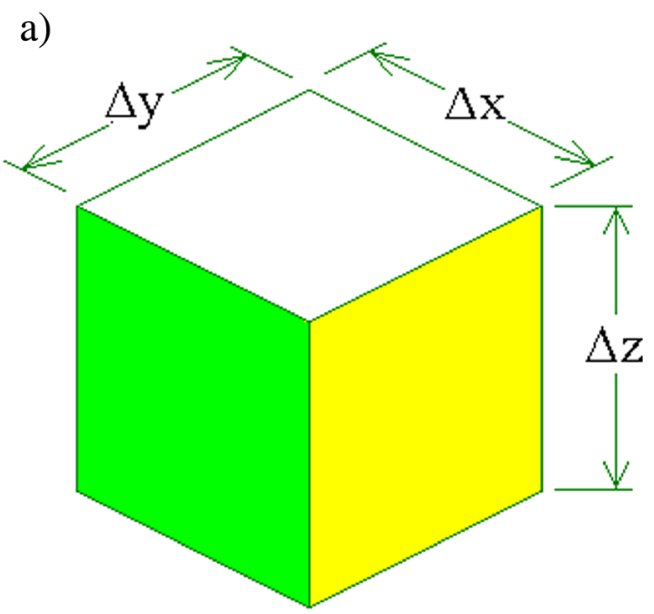

b)

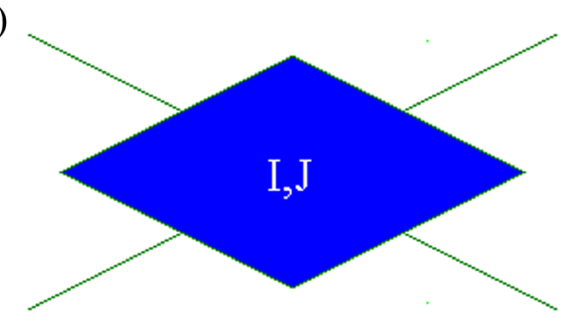

c)

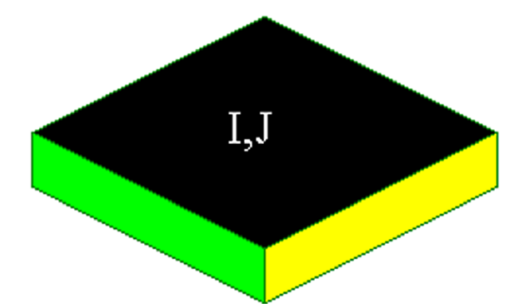

Fig. 1 Data management a) 3D element node used for discretization of the control volume for the steel during the continuous casting. b) Elementary 2D node to solve steel thermal behavior. c) Cell used to display the cellular automata on the computer screen

how the steel thermal behavior can be re-built using only the evolution time information.

$\frac{\mathrm{H}_{\mathrm{I}, \mathrm{J}}^{\mathrm{t}}}{\mathrm{H}_{\mathrm{sol}}-\mathrm{H}_{\text {liq }}}=\frac{\mathrm{T}_{\mathrm{I}, \mathrm{J}}^{\mathrm{t}}}{\mathrm{T}_{\mathrm{sol}}-\mathrm{T}_{\text {liq }}}=\frac{\mathrm{t}}{\mathrm{t}_{\text {solI }, \mathrm{J}}-\mathrm{t}_{\text {liq } \mathrm{I}, \mathrm{J}}}$

\section{Working data}

The simulation done in the present work takes as working data the information saved during previous steel thermal behavior [24]. This model was developed to calculate the heat removal on steel billets produced by continuous casting. A routine for saving the solidification times was nested on the original algorithm at the end of the thermal calculation and displaying procedures. This routine is shown in Fig. 2. Here the pair of loops are executed to compare the temperature on each node 


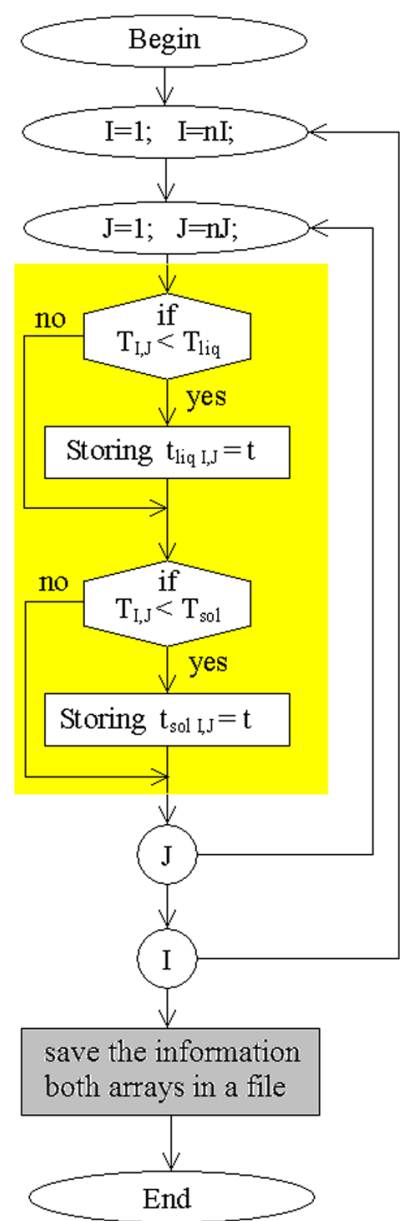

Fig. 2 Flowchart of the routine to save the solidification times during heat transfer simulation on $\mathrm{CCP}$

$\left(T_{I, J}\right)$ with the transition temperatures $\left(T_{\text {liq }}\right)$ and $\left(T_{\text {sol }}\right)$; then if anyone of the sentences are verified as true, the value of the actual time $(t=t+\Delta t)$ is stored in the corresponded computational arrays $\left(\mathrm{t}_{\text {liq I,J }}\right)$ or $\left(\mathrm{t}_{\mathrm{sol}} \mathrm{I,J}\right)$. These arrays are saved at the end of the simulation using independent files.

The main assumption is that every nodal position has a nodal energy $\left(\mathrm{H}_{\mathrm{I}, \mathrm{J}}\right)$ corresponded with a temperature $\left(\mathrm{T}_{\mathrm{I}, \mathrm{J}}\right)$ during simulation. Therefore the times $\left(\mathrm{t}_{\mathrm{liq}} \mathrm{IJ}, \mathrm{J}\right)$ or $\left(\mathrm{t}_{\mathrm{sol}} \mathrm{I,J}\right)$ are the times when each node changes from liquid to mushy and from mushy to solid. Thus it is possible to affirm that the Eq. (1) is valid and the result is known as solid fraction of each analyzed node at any step time.

A routine to save the general information about the cast billet was included in order to make compatible this information with other models. The general information includes the identification of the geometry of the cast section using a numerical code for recognition, then the billet sizes (lx) and (ly), the casting speed ( $\mathrm{v}_{\text {casting }}$ ), steel composition, the transition temperatures $\left(\mathrm{T}_{\text {liq }}, \mathrm{T}_{\mathrm{sol}}, \mathrm{T}_{\mathrm{AR} 1}, \mathrm{~T}_{\mathrm{AR} 3}\right)$, the value of $(\Delta \mathrm{z})$ and step time $(\Delta \mathrm{t})$ are also stored using a floating point data type. Then the nodes used for discretization (nI) and (nJ) are saved using integer data type. These are the elementary data and information to identify every casting case. The size of the elements used for discretization are not saved due to these can be calculated using Eqs. (2) and (3) respectively.

$\Delta \mathrm{x}=\frac{\mathrm{lx}}{\mathrm{nI}}$

$\Delta \mathrm{y}=\frac{\mathrm{ly}}{\mathrm{nJ}}$

The values of (nI) and (nJ) are the upper limits for every loop will be run for analyzing and displaying grain growth. In the same way, the sub indexes (I) and (J) are used to identify each nodal position on the array. The flowchart on Fig. 3 shows the process described. Here the general information is read in the header. Then the filenames where the arrays were saved are read. Immediately a pair of loops is executed to load each nodal value. The times every node remain in mushy $\left(\mathrm{T}_{\text {mushy I,J }}\right)$ are not saved due to these values can be obtained executing a pair of loop for each nodal position (I) and (J) and solving Eq. (4) for each one.

$\mathrm{t}_{\text {mushyI }, \mathrm{J}}=\mathrm{t}_{\text {solI }, \mathrm{J}}-\mathrm{t}_{\text {liqI }, \mathrm{J}}$

\section{Increasing resolution (array size)}

A squared mesh with regular structured element was used to discretizate the steel control volume; a squared mesh with $(100 \times 100)$ nodes gives a very good approach for the steel thermal behavior of the billet. Nevertheless it is necessary to increase the nodes used for discretization in order to obtain a cellular automata with a better resolution to be displayed on the screen. The flowchart on Fig. 4 is used to generate a new improved mesh. The procedure in the shaded area is nested in the analysis loops. This procedure is applied to both computational arrays saved. Here (f) is the increasing factor. (f) is an integer and it must be greater than 1 . Calculation of the new nodal values is obtained solving the Eqs. (5) to (7).

$t_{i}=t_{I, J}-i \frac{\left(t_{I, J}-t_{I+1, J}\right)}{f}$

$\mathrm{t}_{\mathrm{i}}=\mathrm{t}_{\mathrm{I}+1, \mathrm{~J}}-\mathrm{i} \frac{\left(\mathrm{t}_{\mathrm{I}, \mathrm{J}+1}-\mathrm{t}_{\mathrm{I}+1, \mathrm{~J}+1}\right)}{\mathrm{f}}$

$t_{i, j}=t_{i},-j \frac{\left(t_{i}-t_{i}^{\prime}\right)}{f}$

The new nodal corresponding positions (ii, $\mathrm{jj}$ ) are calculated solving the equations inside the flowchart. The new array is saved in an independent file and the new nodal positions will 


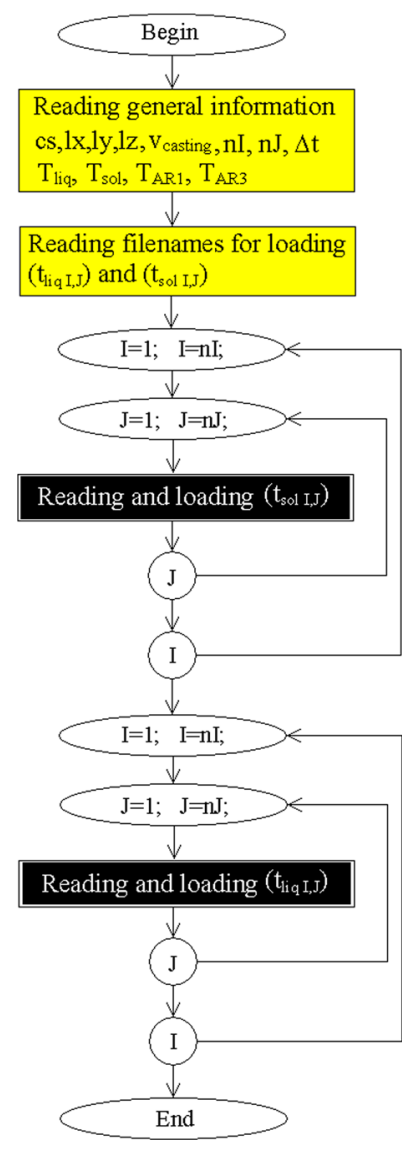

Fig. 3 Flowchart of the routine to read the information about the solidification process

be returned and then identified as (I) and (J) normally positions when the routine for the simulation of the grain structure routines is executed.

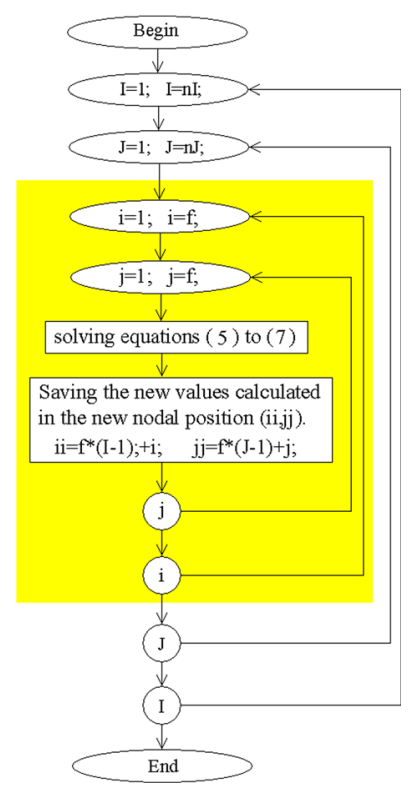

Fig. 4 Flowchart of the procedure to increase the arrays size
Equations (5) to (7) involve the analyzing node $\left(t_{\mathrm{I}, \mathrm{J}}\right)$ and the 3 nearest neighbors which form a singled analyzed group. From these a new set of sub nodes are calculated as is described in Fig. 5. Here a new set of 25 new nodes can be obtained from an original singled group if an increasing factor ( $f=5$ ) is used. The dark nodes are the initial group and the white nodes are the new calculated nodes. This procedure will provide more nodes and a better resolution allowing the simulation of the heterogeneity of the grain morphology in a better detail.

\section{Steel thermal behavior displaying}

A reconstruction of the steel thermal behavior can be done using the information saved on the arrays. The flowchart on Fig. 6 shows the procedure to identify if a node is in liquid, in mushy or solid state as the simulation time goes. This routine is nested on a time simulation loop and produces a 3D full body of the cast billet according with the solidification times as is shown in Fig. 7a to c. Here the steel solidification can be appreciated dynamically. These figures were run inversely with a $(1 / 2)$ cut view used to see the internal evolution of the steel solidification.

Appropriated and logical instructions, loops and routines must be programmed to display the steel solidification. The loops limits for the (1/2) cut view are shown in Fig. 7a to $\mathrm{c}$ are described next:

From $(I=1)$ to $(I=n I)$. And From $(J=1)$ to $(J=n J / 2)$.

These loops must be executed in logical order nesting the loop for (I) inside the loop for (J). Moreover others loops and sentences can be programmed to display different cut views. For a $(1 / 2)$ diagonal cut view a loops Form $(I=1)$ to $(\mathrm{I}=\mathrm{nI})$ must be executed. But $(J)$ is initialized as $(J=1)$ then $(J)$ is incremented at the same time, and the loop is always finished to $(J=n J)$. Other possible option is to declare $(J=I)$ as lower limit and execute the loop from this value to $(J=n J)$.

The main assumptions for all the simulation shown in this work are the following:

Simulation begins at $(t=0)$ and is finished until the latest node is solidified at $\left(\mathrm{t}=\mathrm{t}_{\max }\right)$.

Every node at the begging of the simulation is at the same casting temperature $\left(\mathrm{T}_{\mathrm{I}, \mathrm{J}}=\mathrm{T}_{\text {casting }}\right)$.

The step time $(\Delta t)$ used for simulation is the same than that used during the heat removal calculation.

The simulation is over when the latest node is solidified $\left(t=t_{\max }\right)$. The flowchart shown in Fig. 8 is used to calculate it. $\left(\mathrm{t}_{\max }\right)$ is obtained by comparison between all the solidification times on the arrays. Originally it is 
Fig. 5 Increasing resolution, creating a new set of nodes for increasing the original array size

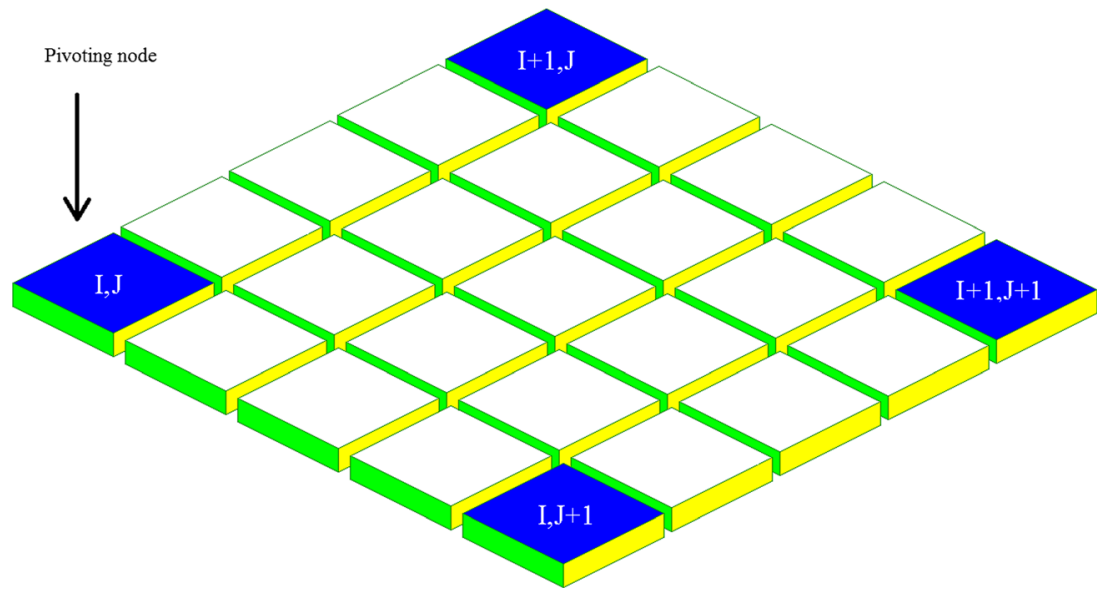

initialized as equal to zero and the value is updated while the sentence "if" continues being verified as true.

The simulation presented in this work correspond to a 1020 carbon steel squared section $(140 \times 140 \mathrm{~mm})$ which was cast

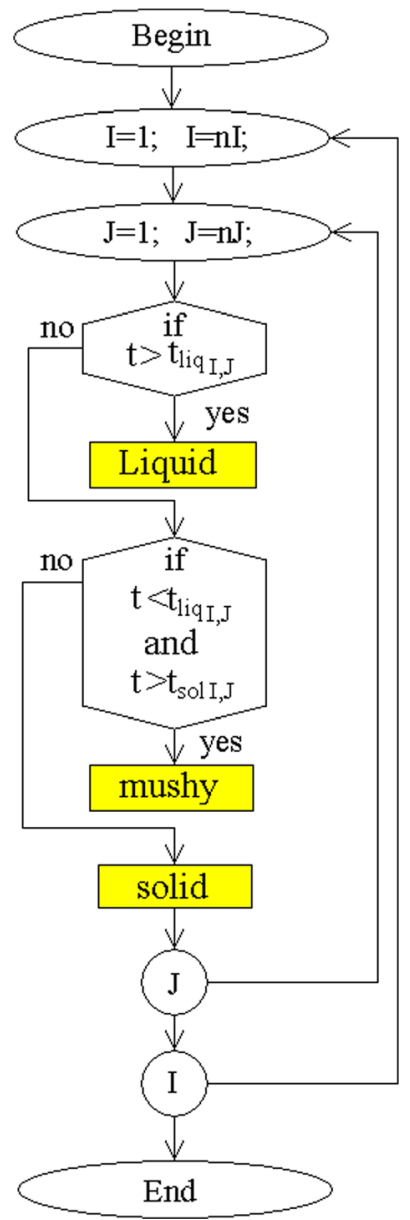

Fig. 6 Flowchart to create a 3D reconstruction of the steel solidification; this routine must be applied after the resolution increment has been executed for a better re-build of the thermal history at $2.55 \mathrm{~m} / \mathrm{min}$. And produced under the quenching conditions shown in Table 1 . A pair of $100 \times 100$ computational arrays were used to store and save the information during the heat removal calculation and an increasing factor $(f=5)$ was used during the dynamic simulation of the solidification.

\section{Grain structure}

Three different grains structures are formed in steel billet produced by CCP according with heat removal conditions. The first is the chill zone. This zone is formed when the steel billet is in the mold. It is a narrow zone over the billet lateral surfaces. It is formed at the begging of the CCP; and must be strong enough to support the internal liquid steel in the core. Here a great number of nucleation points are solidified quickly as a consequence of the high solidification speed. Nucleation is the dominant solidification mechanism. Here the nodes pass from liquid to solid almost instantly and remain only a brief instant in mushy. Then the chill structure is formed with little grains which growth process is blocked by the grains neighbors which are growing simultaneously.

Columnar zone is the second zone. Here the solidification speed has been reduced. The nodes remain here a few seconds in mushy. But the neighbors to the solidified nodes remain with a high solid fraction. On this zone, nucleation and growth process are involved. On the interface between the chill and columnar zones, many nodes are solidified; in consequence these nodes can not growth with lateral neighbors due to these are being solidified simultaneously but there are neighbors with high solid fractions in a perpendicular direction to these. This fact promote that the solidified nodes grow in an opposite direction to the heat removal flux but also perpendicular to the nearest billet surfaces. Thus a columnar grain morphology is resulted.

Finally, inside the billet core the nodes remain a long time in mushy. The solidification speed is very slow due to the heat must be conducted across the previously solidified wall nodes 
a)

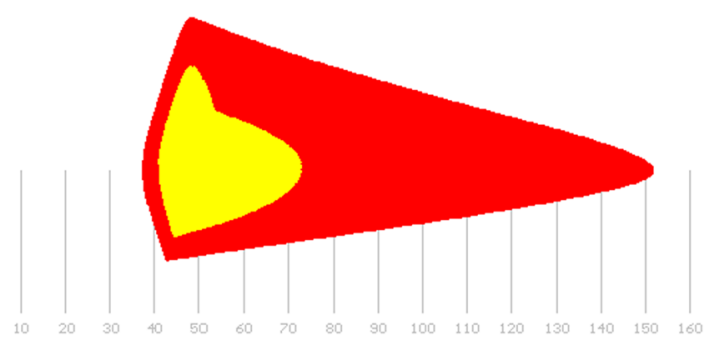

b)

Time (s)

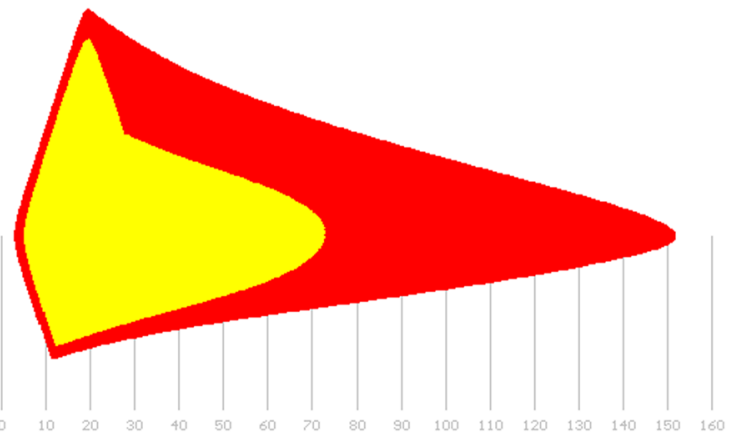

Time (s)

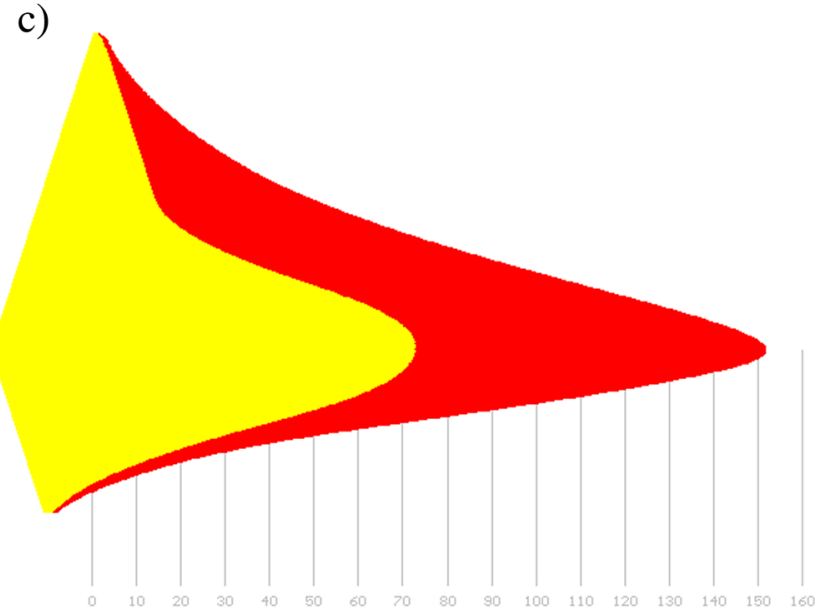

Time (s)

Fig. 7 Inverse 3D display formed with array information for the steel solidification using times information $\left(t_{\mathrm{I}, \mathrm{J}}\right)$; with a $1 / 2$ cut view along the cast direction which allows to watch the steel thermal behavior inside the billet core

to be removed. Here nucleation and grow mechanisms work together to form a zone with big equiaxed grains.

The solidification times can be used to establish a criterion to limit the sizes of the chill, columnar and equiaxed zones. $\left(\mathrm{t}_{\mathrm{CCT}}\right)$ and $\left(\mathrm{t}_{\mathrm{CET}}\right)$ are the time criterions to the chill-columnar and columnar-equiaxed transition zones. These values are compared with the nodal times $\left(t_{I, J}\right)$ to be classified according the simulation time $(t+\Delta t)$. The best fit for the zones morphology was obtained using the following values $\left(\mathrm{t}_{\mathrm{CCT}}=2 \mathrm{~s}\right)$ and $\left(\mathrm{t}_{\mathrm{CET}}=12 \mathrm{~s}\right)$ and comparing with the mushy times $\left(\mathrm{t}_{\text {mushy I,J }}\right)$.

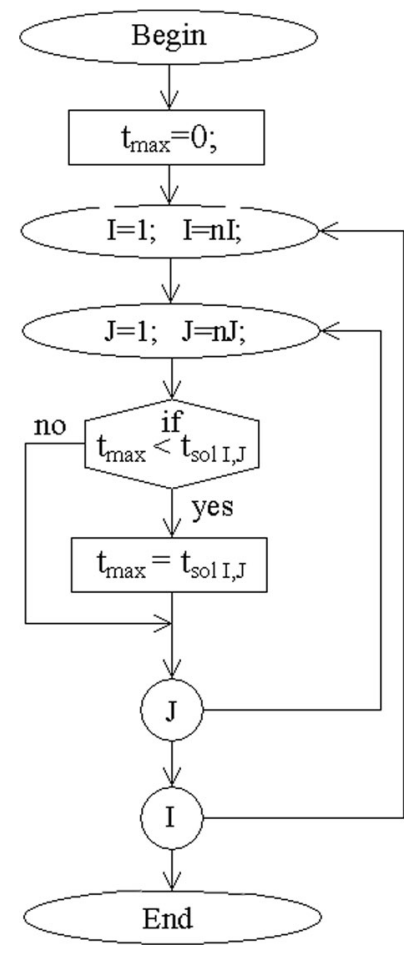

Fig. 8 Flowchart to find the maximum solidification time $\left(t_{\max }\right)$

The evolution of solidification can be analyzed counting the nodes in liquid, mushy and solid in order to establish mathematical and physical criterions and assumptions to reproduce the primary grain structure. The flowchart on the Fig. 9 shows the logical procedure to classify and count the nodes in liquid and solid state. This routine can be executed at each simulation time $(\mathrm{t}+\Delta \mathrm{t})$. The variables $\left(\mathrm{nn}_{\text {liq }}^{\mathrm{t}}\right)$ and $\left(\mathrm{nn}_{\text {sol }}^{\mathrm{t}}\right)$ are used for counting the nodes on the corresponding state. These values are always initialized as equal to zero. Within, the pair of loops used for analysis is executed. Then the values of the variables $\left(\mathrm{nn}_{\text {liq }}^{\mathrm{t}}\right)$ and $\left(\mathrm{nn}_{\mathrm{sol}}^{\mathrm{t}}\right)$ are increased if the sentences are verified as true.

The flowchart on the Fig. 10 shows the logical procedure to classify and count the nodes on mushy. Here the variable $\left(\mathrm{nn}_{\text {mushy }}^{\mathrm{t}}\right)$ is also initialized as equal to zero and it is increased only if the both sentences are verified as true. In the same way than the algorithm on Fig. 9 this routine can be executed at each simulation time.

Figure 11a to $\mathrm{c}$ show the three different classifications for the values of the solidification times. These figures were resulted from the application of the algorithms described on Figs. 9 and 10. Figure 11a shows a classification obtained after counting all the nodes on liquid and solid state during the simulation. These curves represent the total nodes changing from liquid to mushy and from mushy to solid at each step time. These curves are initialized as equal to zero due to all the nodes are at the same temperature $\left(\mathrm{T}_{\text {casting }}\right)$ in liquid state when the simulations begins $(t=0)$. Then the nodes on the billet surface begin to be solidified. The vertical slope of these 
Table 1 Operating conditions used on the secondary cooling system for the casting of steel 1020

\begin{tabular}{|c|c|c|c|c|c|c|c|c|c|}
\hline \multirow{2}{*}{$\begin{array}{l}\text { Case } \\
\text { Segment }\end{array}$} & \multicolumn{4}{|l|}{1} & \multicolumn{2}{|l|}{2} & \multicolumn{3}{|l|}{3} \\
\hline & 1 & 2 & 3 & 4 & 1 & 2 & 1 & 2 & 3 \\
\hline Water flow rate $(1 / \mathrm{min})$ & 192 & 250 & 210 & 210 & 175 & 148 & 107 & 220 & 147 \\
\hline Sprays & 1 & 9 & 9 & 9 & 10 & 10 & 8 & 11 & 8 \\
\hline $\mathrm{D}_{\mathrm{bs}}(\mathrm{mm})$ & 160 & 160 & 160 & 160 & 150 & 150 & 60 & 110 & 75 \\
\hline
\end{tabular}

graphs on the initial $10 \mathrm{~s}$ indicates a high solidification speed. Here the horizontal distance between the two curves is very short; this means that the nodes being solidified remain only a brief time in mushy. The nodes which suffer this hard transition are those near the billet surface. Nevertheless, as the simulation continues the slopes tend to decrease until be almost horizontal; this is an evidence of the reduction on the solidification speed. But the horizontal distances between both curves are increased; thus these nodes remains longer times in mushy. It can also be appreciated that the longest residence time in mushy is when all the nodes $(100 \%)$ have been passed from liquid to mushy (74 s.) for the analyzed case.

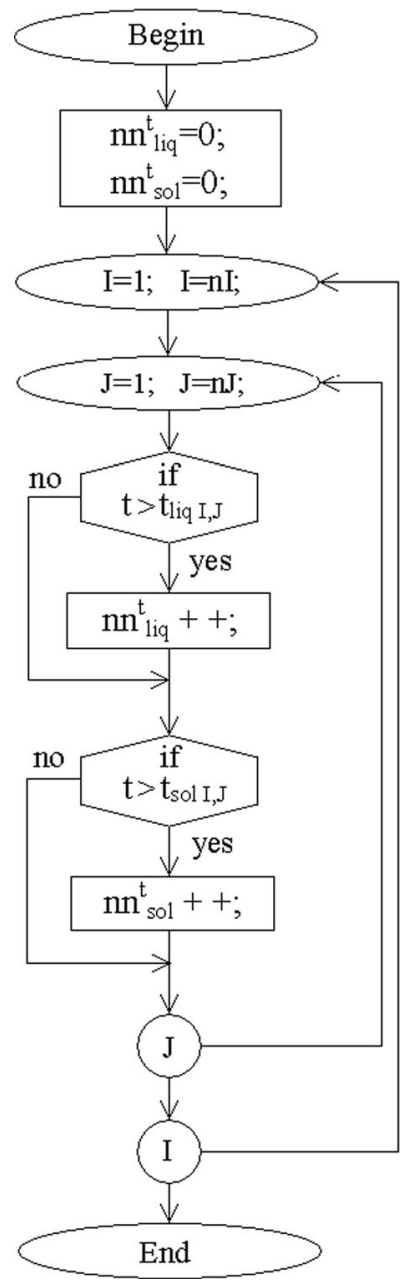

Fig. 9 Flowchart for classifying and counting nodal times according with physical state (liquid and solid)
The curve in Fig. 11b represents the nodes in mushy at each simulation time during the simulation. This curve is graphed counting all the nodes in mushy. This curve geometrically means the measurement of the horizontal distance described in Fig. 11a. This curve also begins equal to zero; but a quick increment on the solidified nodes can be observed. In consequence the slope is also increased to be almost vertical. After $10 \mathrm{~s}$ the slope tends to decrease with a moderate behavior. Nevertheless the nodes in mushy continues being incremented until a maximum value. This value is on $74 \mathrm{~s}$; the same time, than that when all the nodes have been passed from liquid to mushy. Finally the curve tends to be reduced until be equal to zero due to at the end of the simulation there are no nodes on mushy because all of these have been solidified. This curve is also an evidence of the facts previously mentioned and reinforces the relation between the mathematical validations for the physical principles used to solve the computational model during the calculation of the heat removal.

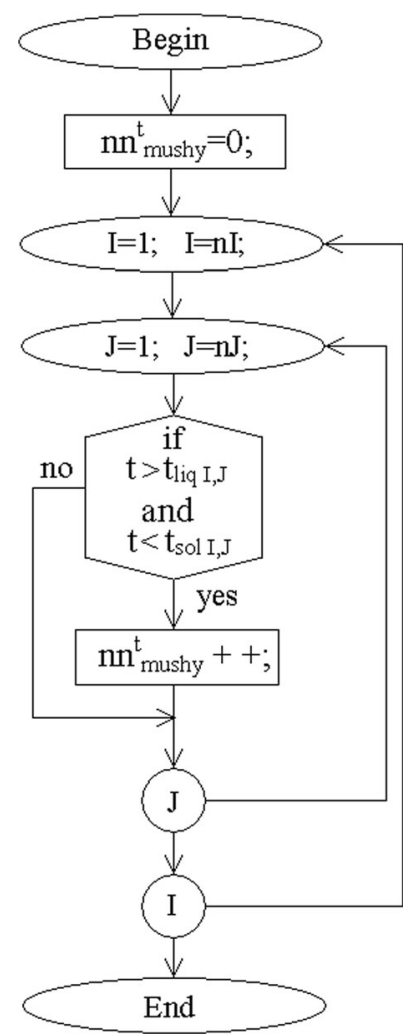

Fig. 10 Flowchart for classifying and counting nodal times in mushy 
a)

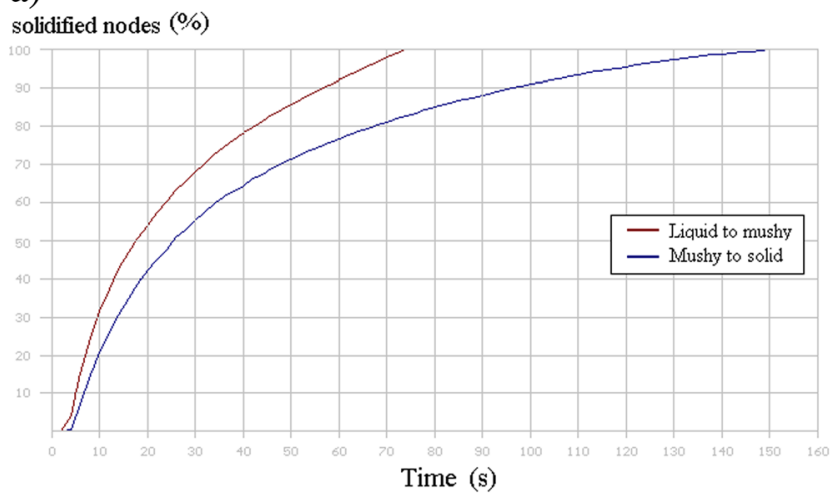

b)

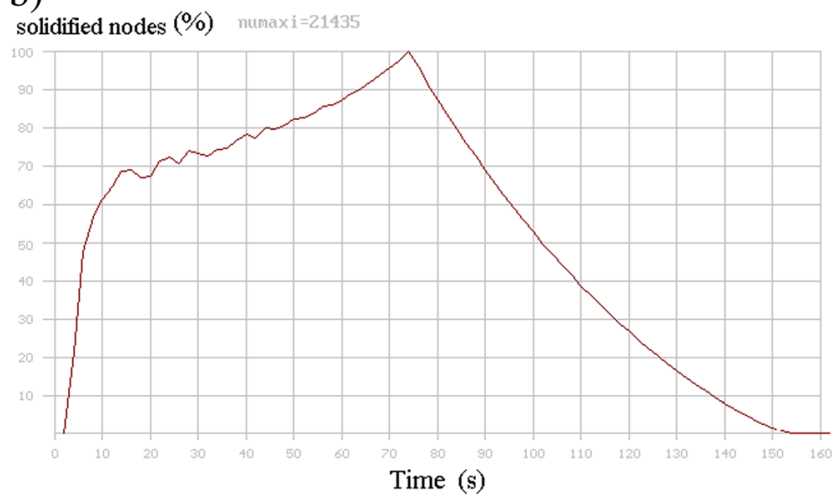

c)

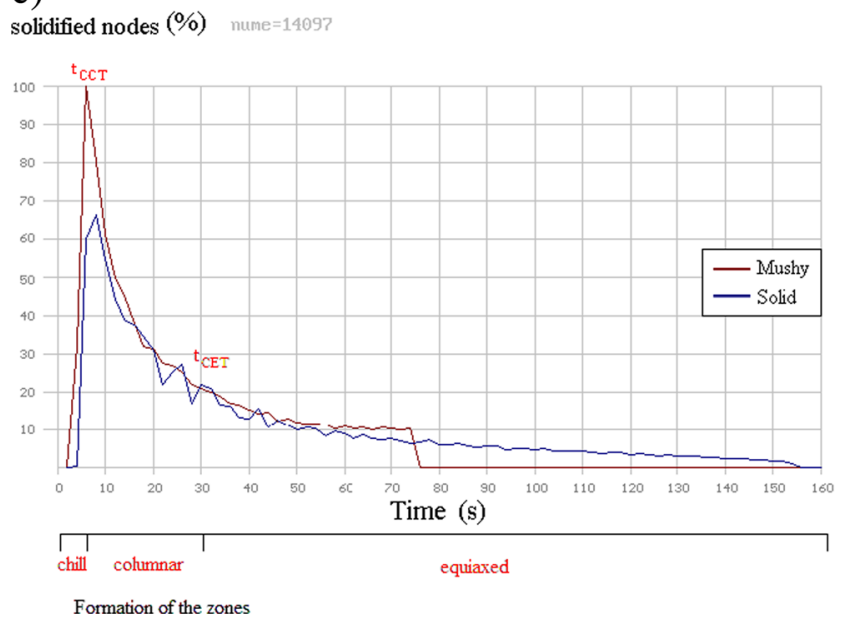

Fig. 11 Classification of the solidification times a) curves for total nodes on $\left(t_{\text {liq I,J }}\right)$ and $\left(t_{\text {sol I,J }}\right) . b$ ) curve for total nodes in mushy. c) curves for instantaneous solidification speeds nodes changing from liquid to mushy and from mushy to solid

The curves shown in Fig. 11c are called instantaneous speeds. These curves are the nodes in liquid and solid state at each step time. Both of these curves also are initialized as equal to zero. And these slopes are increased strongly due to the temperature has been reduced quickly in the nodes near the billet surface. In other words the number of solidified nodes (a big population) is quickly increased until a maximum value. Then the nodes being solidified are quickly reduced. Then, the slopes tend also towards a horizontal behavior. The first curve on returning to zero is which represent the nodes being transferred from liquid to mushy. This fact is also appreciated here. All these 3 figures are evidence of the solidification speed influence on the grain structure will be formed.

\section{Nucleation and grain growth}

The formation of a primary grain structure is due to the solidification process after foundry steel. New grains appear as the solidification front advances. Simulation of the solidification in metallic materials involves the representation of nucleation and grain growth processes. These processes must be logically programmed according with the evolution of the solidification in order to represent dynamically the grain formation (as a function of the simulation time). The assumptions to simulate nucleation and grain growth processes are the following:

There are no solidified nodes at the beginning of the simulation $(t=0)$. Therefore all the cells are returned to an initial value $\left(c_{I, J}=0\right)$. In other words, all the cellular automata cells are cleaned and returned to zero for each new simulation.

When a node has been solidified only one mechanism of solidification will be applied (nucleation or growth).

Solidification state in cellular automata is a permanent status. Then a solidified node can not be returned to mushy or liquid state.

When a node has been solidified the initial value on the cellular automata is defined with a numerical value in consequence $\left(c_{I, J} \neq 0\right)$.

A node is naturally solidified if its solidification time is equal than the simulation time $\left(t=t_{\text {sol I,J }}\right)$. Then a random number is generated to decide if the node is nucleated or is growth.

If the solidified node is nucleated; a random number is generated to define the numerical code for the new nucleated node; this value is assigned to the corresponding cell and a pixel is displayed on the screen.

If the solidified node is growth a neighboring analysis is executed in order to decide which neighbor will grow with.

All the decisions about if a node is nucleated or growth are taken including the generation of an integer random number and compared with probabilities as is done in Monte Carlo Methods [6-8].

The model was compiled to create a two dimensional cellular automata. Therefore the 8 nearest surrounding neighbors are consider for growing. Nevertheless a previous analysis is executed to know which of these are available for growing.

An available neighbor must be part of the computational array. According with this in Fig. 12a to $\mathrm{c} \mathrm{some} \mathrm{nodes} \mathrm{over} \mathrm{the}$ billet surface will have only 5 available neighbors and nodes in the corners will have only 3 available neighbors. 

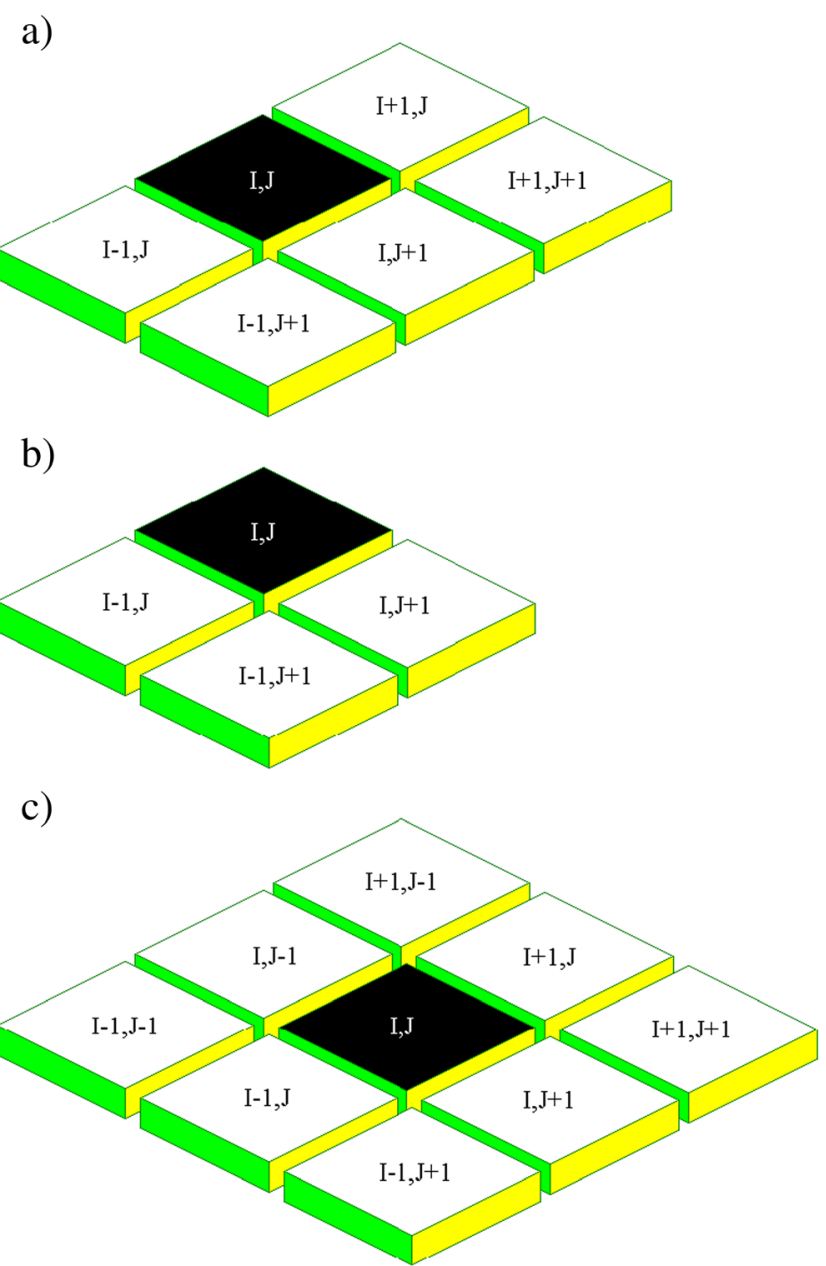

Fig. 12 Available neighbors for analyzing nodes in the billet. a) on the billet lateral surfaces. b) on the billet corners. c) on every nodal position inside the billet

A solidified node inside the array will have less than 8 available neighbors if some of the neighbors have been previously solidified.

Nucleation and growth process are executed as a function of the solidification speed. During the formation of the chill zone there are many nucleated grain appearing on the billet surface. Here, the dominant mechanism is nucleation. In columnar zone, the nucleation decreases and growth process is promoted due to the neighbors with high solid fractions which tend to coalesce with the previously nucleated nodes and a columnar straight growing direction is promoted. Finally in the equiaxed zone the nodes remain long times in mushy then the probability to grow is distributed with all the available neighbors.

In addition, the present algorithm includes the presolidification process. It is consider that a node can be presolidified if the node is in mushy with a high enough solid fraction $\left(\mathrm{X}_{\mathrm{sol} I \mathrm{JJ}}\right)$. Nevertheless a node on liquid can not be consider for pre-solidification.
Solid and liquid fractions are calculated at each step time during simulation for every node using the Eqs. (8) and (9) respectively. Here the second term on Eq. (8) is solved when the steel thermal behavior is being calculated. Thus this equation is a function of the transformation and actual temperatures. The third term is solved during simulation in the present work due to the values of the arrays are times.

$X_{\text {solI }, J}^{t}=\frac{T_{\text {sol }}-T_{I, J}}{T_{\text {sol }}-T_{\text {liq }}}=\frac{t_{\text {soli }, J}-t}{t_{\text {solI }, J}-t_{\text {liqI }, J}}$

$\mathrm{X}_{\text {liq }}^{\mathrm{t}}=1-\mathrm{X}_{\mathrm{sol}}^{\mathrm{t}}$

\section{Computational algorithm}

The main algorithm used to simulate grain formation dynamically is shown in Fig. 13. Here the zone where each node

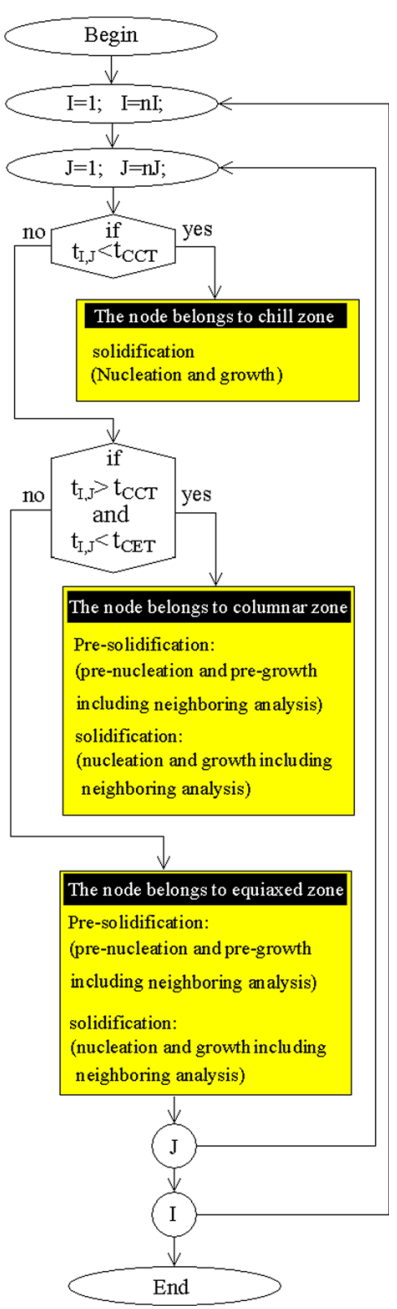

Fig. 13 General flowchart to simulate nucleation and grain growth procedures 
belongs (chill, columnar or equiaxed) is identified by comparison. Then routines for solidification and pre-solidification are executed. The criterions $\left(t_{\mathrm{CCT}}\right)$ and $\left(\mathrm{t}_{\mathrm{CET}}\right)$ are usually established according with mushy times $\left(t_{\text {mushy } I, J}\right)$ as is was explained; nevertheless new criterions can be established using the classification exposed in this work [1-15].

The points shown in Fig. $11 \mathrm{~b}$ and $\mathrm{c}$ represents the maximum number of solidified nodes during the simulation [22-24]. Here the averaged mushy time of the nodes being solidified is $(2.2 \mathrm{~s})$. This value can be established as a criterion for transition from chill to columnar. It is important to mention that curves on Fig. 11b and c are not constant due to the step time selected was $0.5 \mathrm{~s}$ which is different to the value used during the heat removal calculation was $0.02 \mathrm{~s}$.

In Fig. 13; if the first sentence is verified as true, the node is in the chill zone. Here the predominant mechanism is nucleation. The grain growth process is blocked by the nodal neighbors being solidified at the same time. Here the nucleation and growth probabilities are 50-50. The result is a not wide fine structure formed with little grains.

If the first sentence is false the value of the time is compared with the second criterion. Then if both sentences are verifies as true, the node belongs to the columnar zone and the routines for nucleation and growth are executed. Here the probabilities for growing are greater than those for nucleation [22]. The nodes in this zone remain almost $8 \mathrm{~s}$. in mushy; this value is other important criterion that must be defined. Then preferential growth directions are assigned to the available neighbors according with the fraction solids and solidification times. The shortest time difference is calculated using the Eq. (10). Here the sub indexes (i) and (j) are used to identify the neighbors. Then all the values calculated are stored temporally to be compared in order to find the shortest; which will be the most probable node to be solidified. The entire verification procedure for checking the availability of a node is described in the flowchart of Fig. 14. Here (nn) is the number of available nodes.

$\mathrm{dt}_{\text {prei }, \mathrm{j}}=\mathrm{t}_{\text {solI }, \mathrm{J}}-\mathrm{t}_{\text {soli, }}$

Finally if the two previous comparisons are false the node belongs to the equiaxed zone. Here the nucleation process is reduced and the preferential mechanism is turned towards growth; due to the neighbors are with a constant decrement on the high solid fractions as a function of a reduction of the solidification speed.

If a node is pre-solidified, means that the solidification has been accelerated artificially by the algorithm. The presolidified nodes get down its nodal temperature until $\left(\mathrm{t}_{\mathrm{sol}} \mathrm{I,J}\right)$. in consequence the latent heat must be redistributed with the nearest un-solidified neighbors (nun). Then it is necessary to execute an additional neighboring analysis to find these.

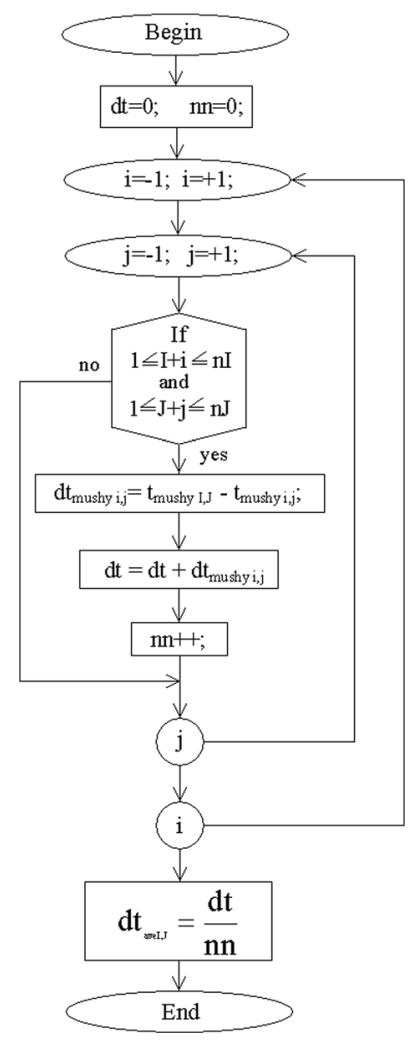

Fig. 14 Flowchart for neighboring analysis

Heat redistribution is done assuming a homogeneous conduction of the energy. Then an increment on the neighbor's nodal temperature is resulted. Thus the solidification times will be also increased. The Eq. (11) must be solved to know the time difference to be distributed when a node has been pre-solidified.

$\mathrm{dt}_{\text {preI }, \mathrm{J}}=\mathrm{t}_{\text {solI }, \mathrm{J}}-\mathrm{t}$

Here $(\mathrm{t})$ is the simulation time at which the node was solidified. Then Eq. (12) is used to know new solidification times for the neighbors. Here the first term in left is the time will be sum to the available neighbors. Then the new calculated times are temporally stored in an independent updated array used only for the simulation of this case.

$\mathrm{t}_{\text {solI }, \mathrm{J}}=\frac{\mathrm{dt}_{\text {preI }, \mathrm{J}}}{\text { nun }}+\mathrm{t}_{\text {solI }, \mathrm{J}}$

\section{Simulations}

Figure 15 shows a grain structure obtained using the model developed. Here a squared billet is displayed and is possible to appreciate the 3 different grain structures. In the billet surfaces 


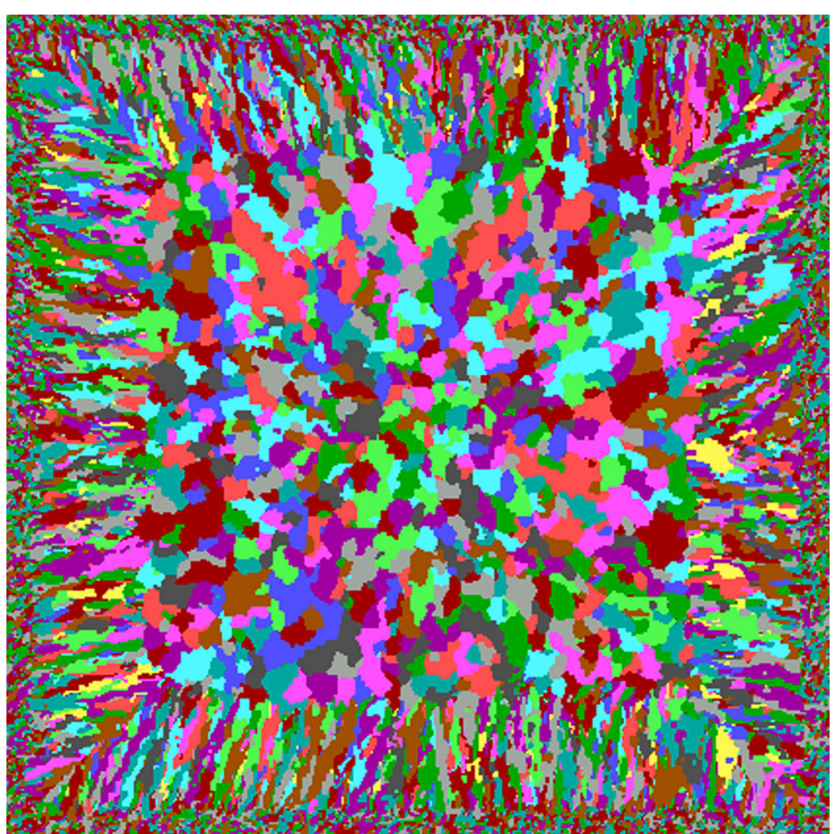

Fig. 15 Grain structure simulated (including chill, columnar and equiaxed zones in a squared billet)

the chill zone is well defined. Small grain sizes are here due to the high solidification speed. The transition between chill and columnar zones is rounded near the corner due to these are the coolest regions. The reason is because the relation between the area where heat was removed and heat flux is greater in the corners than in the middle surfaces of the billets.

There is a columnar grain morphology as the solidification speed is reduced. Near the corners columnar grains begin to grow but its growth is interrupted with the grain growing in the perpendicular direction; reason why the preferential growth direction in this zone is against the heat removal flux and perpendicular to the nearest billet surface; and the probabilities of the available neighbors for growing are increased inversely to the time difference calculated with Eq. (11). Moreover it is possible to affirm that columnar nodes are dynamically formed due to the nucleation and growth of the nodes in the border of the solidification front interrupt their lateral growth promoting a preferential growth direction against heat removal flux.

In the billet core there is a zone with equiaxed grains formed due to the long time these nodes remain in mushy. In consequence a nucleated node will tend to growth with all the available neighbors. Here there is no a preferential growth direction as was shown in columnar zone due to the time differences calculated with Eq. (11) are very close.

The Fig. 16a-c are from a real sample of a steel billet produced by continuous casting and the similarity with the grain structure simulated is notorious. Figure 16a is a photo taken near the billet surface her it is possible to observe chill and columnar structures. Figure $16 \mathrm{~b}$ was taken from a corner here it is possible to observe chill and columnar zones again; but
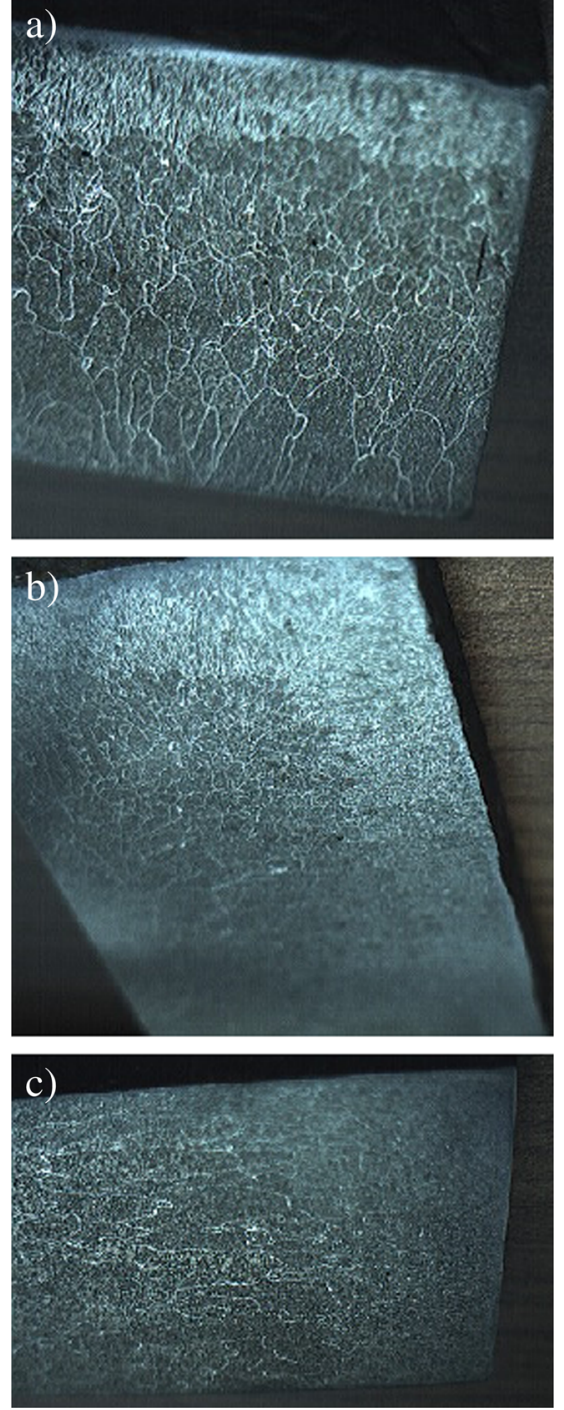

Fig. 16 Grain structure obtained from a real billet produced by continuous casting. b) Photo taken in the corner. b) Photo taken near the billet surface. c) Photo taken in the billet core

also the perpendicular effect of the heat removal on the columnar gains. Finally Fig. 16c shows very big grains from the equiaxed central zone inside the billet core.

\section{Conclusions}

Geometrical form of the curves for solidification speed, indicate the strong influence on the solidification mechanisms involved nucleation and grain growth. Moreover the criterions based on the time classification $\left(\mathrm{t}_{\mathrm{CCT}}\right)$ and $\left(\mathrm{t}_{\mathrm{CET}}\right)$ reinforce this evidence and confirm these values can be employed to establish the limits between the grain zones.

The change on the slopes and areas under the curves for the classification of nodes evidences mathematical physically the 
influence of the solidification times and speeds over the grain structure resulted.

Grain morphology on the zones chill, columnar and equiaxed was very appropriately simulated with the inclusion of the nucleation and grain growth procedures.

The solidification process was successfully simulated and displayed on the screen dynamically.

This model demonstrated that the information used as input (resulted from a deterministic numerical model) can be employed to re-build the thermal story and also can be used with stochastic procedures to obtain a very close approaching representation of the grain structure.

The use of nested procedures, sub routines and loops provide an efficient simulation model and the unnecessary code was properly eliminated.

The generation of a new improvement mesh was also successfully done and proved that a better resolution array for representing the grain structures can be generated.

Nucleation is the predominant mechanism at the beginning of the simulation due to high solidification speed; but has this speed decreases the growth becomes stronger.

Acknowledgments The authors want to thanks to the institutions: Consejo Nacional de Ciencia y Tecnología (CONACyT), Universidad Autónoma Metropolitana (UAM-Azc), Instituto Tecnológico de México (ITAM) with special mention to Asociación Mexicana de Cultura A.C.

Open Access This article is distributed under the terms of the Creative Commons Attribution 4.0 International License (http:// creativecommons.org/licenses/by/4.0/), which permits unrestricted use, distribution, and reproduction in any medium, provided you give appropriate credit to the original author(s) and the source, provide a link to the Creative Commons license, and indicate if changes were made.

\section{References}

1. Flemings MC (1974) Solidification Processing, New York Ed., McGraw Hill 288 Book Co.

2. Lan YJ, Li DZ, Li YY (2004) Modeling austenite decomposition into ferrite at different cooling rate in low-carbon steel with cellular automaton method. Acta Mater 52:1721-1729. doi:10.1016/j. actamat.2003.12.045

3. Zhang L, Zhang CB, Wang YM, Wang SQ, Ye HQ (2003) A cellular automaton investigation of the transformation from austenite to ferrite during continuous cooling. Acta Mater 51:5519-5527. doi:10.1016/S1359-6454(03)00416-6

4. Yee K, Hong CP (1997) Stochastic modeling of solidification grain for structures of $\mathrm{Al}-\mathrm{Cu}$ Crystalline ribbons in planar flow casting. ISIJ Int 37(1):38-46. doi:10.2355/isijinternational.37.38

5. Shin YH, Hong CP (2002) Modeling of dendritic growth with convection using a modified cellular automaton model with a diffuse Interface. ISIJ Int 42(4):359-367. doi:10.2355 /isijinternational.42.359

6. Ivasishin OM, Shevchenko SV, Vasiliev NL (2003) 3D MonteCarlo simulation of texture-controlled grain growth. Acta Mater 51:1019-1034. doi:10.1016/S1359-6454(02)00505-0

7. Mishra S, DebRoy T (2004) Measurements and Monte Carlo simulation of grain growth in the heat-affected zone of Ti-6Al-4 V welds. Acta Mater 52:1183-1192. doi:10.1016/j. actamat.2003.11.003

8. Tong M, Li D (2004) Yiyi Li, modeling the austenite-ferrite diffusive transformation during continuous cooling on a mesoscale using Monte Carlo method. Acta Mater 52:1155-1162. doi:10.1016/j. actamat.2003.11.006

9. Lan CW, Chang YC, Shih CJ (2003) Adaptive phase field simulation of non-isothermal free dendritic growth of a binary alloy. Acta Mater 51:1857-1869. doi:10.1016/S1359-6454(02)00582-7

10. Zhu MF, Kim JM, Hong CP (2001) Modeling of globular and dendritic structure evolution in solidification of an $\mathrm{Al}-7 \mathrm{mass} \% \mathrm{Si}$ alloy. ISIJ Int 41(9):992-998. doi:10.2355/isijinternational.41.992

11. Zhu MF, Hong CP, Modified Cellular A (2001) Automaton model for the simulation of dendritic growth in solidification of alloys. ISIJ Int 41(5):436-445. doi:10.2355/isijinternational.41.436

12. Holm EA, Miodownik MA, Rollett AD (2003) On abnormal subgrain growth and the origin of recrystallization nuclei. Acta Mater 51:2701-2716. doi:10.2355/isijinternational.41.436

13. Yoshioka H, Tada Y, Hayashi Y (2004) Crystal growth and its morphology in the mushy zone. Acta Mater 52:1515-1523. doi:10.1016/j.actamat.2003.11.033

14. McAfee R, Nettleship I (2003) The simulation and selection of shapes for the unfolding of grain size distributions. Acta Mater 51:4603-4610. doi:10.1016/S1359-6454(03)00297-0

15. Wang W, Lee PD, McLean M (2003) A model of solidification microstructures in nickel-based superalloys: predicting primary dendrite spacing selection. Acta Mater 51:2971-2987. doi:10.1016/S1359-6454(03)00110-1

16. Feng W, Xu Q, Liu B (2002) Microstructure simulation of aluminum alloy using parallel computing technique. ISIJ Int 42(7):702707. doi:10.2355/isijinternational.42.702

17. Suwa Y, Saito Y, Onodera H (2006) Phase field simulation of grain growth in three dimensional system containing finely dispersed second-phase particles. Scr Mater 55:407-410. doi:10.1016/j. scriptamat.2006.03.034

18. Mc Fadden S, Browne DJ (2006) Meso-scale simulation of grain nucleation, growth and interaction in castings. Scr Mater 55:847850. doi:10.1016/j.scriptamat.2006.08.001

19. Randle V, Rios PR, Hu Y (2008) Grain growth and twinning in nickel. Scr Mater 58:130-133. doi:10.1016/j. scriptamat.2007.09.016

20. Schwartz AJ, King WE, Kumar M (2006) Influence of processing method on the network of grain boundaries. Scr Mater 54:963-968. doi:10.1016/j.scriptamat.2005.11.052

21. Liu DR, Guo JJ, Wu SP, Su YQ, Fu HZ (2006) Stochastic modeling of columnar-to-equiaxed transition in Ti-(45-48 at\%) Al alloy ingots. Mater Sci Eng A 415:184-194. doi:10.1016/j. msea.2005.09.077

22. Ramírez A et al (2009) Randomly grain growth in metallic materials. Chaos, Solitons Fractals 42:820-825. doi:10.1016/j. chaos.2009.02.011

23. Ramirez-Lopez A et al (2012) A cellular automaton model for simulating grain structures with straight and hyperbolic interfaces. Int J Miner Metall Mater 19(8):699-710. doi:10.1007/s12613-0120616-0

24. Ramirez-Lopez A et al (2010) Simulation factors of the steel continuous casting. Int J Miner Metall Mater 17(3):267-275. doi:10.1007/s12613-010-0304-x

25. Gandin C-A, Desbiolles J-L, Rappaz M, Thevoz P (1999) A threedimensional cellular automation-finite element model for the prediction of solidification grain structures. Metall Mater Trans A 30(12):3153-3165. doi:10.1007/s11661-999-0226-2

26. Zhong $\mathrm{H}$ et al (2016) A thermal simulation method for solidification process of steel slab in continuous casting. Metall Mater Trans B 47(5):2963-2970. doi:10.1007/s11663-016-0660-7 
27. Cheng Ji, Chen-hui Wu, Miao-yong Zhu, Thermo-Mechanical Behavior of the Continuous Casting Bloom in the Heavy
Reduction Process, JOM, First Online 02 August 2016, ,pp 1-9. DOI: $10.1007 / \mathrm{s} 11837-016-2041-8$ 